\title{
Tumor necrosis factor receptor 2 may promote the proliferation and drug resistance of Kapras299 and L428 lymphoma cells via the AKT and WNT/ $\beta$-catenin signaling pathways
}

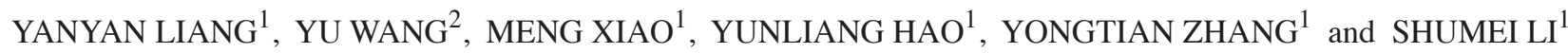 \\ ${ }^{1}$ Department of Hematology, First People's Hospital of Jining City, Jining, Shandong 257335; \\ ${ }^{2}$ Department of Medicine 11, People's Hospital of Xintai City, Tai'an, Shandong 271200, P.R. China
}

Received January 16, 2017; Accepted October 18, 2017

DOI: $10.3892 / \mathrm{ol} .2018 .8396$

\begin{abstract}
Circulating soluble tumor necrosis factor receptor 2 (sTNFR2) has been associated with a relatively poor prognosis in various types of lymphoma. However, the specific role of TNFR2 expression in lymphoma cells remains uncharacterized. In the present study, TNFR2 expression was quantified in the Hodgkin lymphoma cell line, L428, and the anaplastic large-cell lymphoma cell line, Karpas299, using RT-PCR and western blot analyses. Karpas 299 cells exhibited higher TNFR2 expression than L428 cells. Proliferation and drug resistance experiments demonstrated that Karpas299 cells also possessed a greater proliferative ability and resistance to adriamycin (ADM) than L428 cells, with 50\% inhibitory concentrations $\left(\mathrm{IC}_{50}\right)$ of $1.423 \pm 0.24 \mu \mathrm{mol} / 1$ for Karpas 299 cells, compared with $0.728 \pm 0.15 \mu \mathrm{mol} / 1$ for $\mathrm{L} 428$ cells $(\mathrm{P}<0.05)$. The knockdown of TNFR2 in Karpas299 cells significantly reduced their proliferative ability; when treated with ADM, the cell inhibition rate increased from $49.34 \pm 5.42 \%$ to $74.13 \pm 6.81 \%(\mathrm{P}<0.05)$. The upregulation of TNFR2 in L428 cells significantly increased their proliferative ability; when treated with ADM, the cell inhibition rate decreased from $47.03 \pm 5.25 \%$ to $28.71 \pm 4.90 \%(\mathrm{P}<0.05)$. Investigation of the underlying molecular mechanism indicated that the upregulation of TNFR2 expression in L428 cells increased the expression of $\beta$-catenin and the phosphorylation of AKT. In L428 cells overexpressing TNFR2, the $\beta$-catenin blocker, DKK1, or the AKT inhibitor, LY294002, abrogated the increase in proliferation induced by TNFR 2 and increased cell inhibition rate upon treatment with ADM. In summary, the present study demonstrated that TNFR 2 promoted the proliferative and drug resistance abilities of lymphoma cells via
\end{abstract}

Correspondence to: Professor Shumei Li, Department of Hematology, First People's Hospital of Jining City, 6 Health Road, Jining, Shandong 257335, P.R. China

E-mail: lishumeiatjn@126.com

Key words: tumor necrosis factor receptor 2, lymphoma, proliferation, drug resistance, WNT, $\beta$-catenin the AKT and WNT/ $\beta$-catenin signaling pathways. This may provide the experimental basis for the further study of TNFR2 activity in lymphoma cells and warrant its investigation as a therapeutic target for lymphoma.

\section{Introduction}

Lymphoma is a type of malignant tumor derived from the lymph and hematopoietic systems, including the subtypes of Hodgkin lymphoma (HL) and non-Hodgkin lymphoma (NHL) $(1,2)$. Although HL and NHL are all neoplastic diseases of lymphoid tissues, the clinical manifestations and histopathology is in contrast. Reed-Sternberg cells are a characteristic feature of HL and their presence is used for diagnosis. However, in NHL, Reed-Sternberg cells are not utilised for diagnosis. Painless and progressive swelling of lymph nodes is the typical initial symptom of HL; however, extranodal invasion is the common symptom of NHL (3). Due to the high heterogeneity and malignancy of lymphoma, the prognosis remains generally unsatisfactory despite advances in conventional therapies, including radiotherapy, chemotherapy and hematopoietic stem cell transplantation (3-5). The necessity for the development and clinical application of molecularly-targeted drugs has become widely accepted, and the discovery of novel molecular therapeutic targets is essential to improve patient prognosis.

Tumor necrosis factor receptor 2 (TNFR2) is a member of the TNFR family. Previous studies have reported that TNFR2 is mainly expressed in hematopoietic and endothelial cells, with anti-inflammatory (6), protection against lipopolysaccharide-induced lung damage (7) and bone fracture-healing (8) properties. TNFR2 has also been demonstrated to promote the migration and invasion abilities of malignant tumor cells, including cholangiocarcinoma and myeloma cells $(9,10)$. However, the role of TNFR2 in lymphoma cells remains uncharacterized.

Anaplastic large-cell lymphoma (ALCL) is a type of NHL exhibiting CD30 ${ }^{+}$expression. HL is similar to ALCL in cellular morphology, and possesses pathogenic mechanisms analogous to those of ALCL (11). An HL cell line, L428, and an ALCL cell line, Karpas299, were used in the present study to investigate the role of TNFR2 in the proliferation 
and drug resistance of lymphoma cells. A potential molecular mechanism underlying the proliferative and drug resistance functions of TNFR2 was also investigated.

\section{Materials and methods}

Cell lines and cell culture. L428 and Karpas299 cells were obtained from the American Type Culture Collection (Manassas, VA, USA). Both cell lines were cultured in RPMI-1640 medium containing 10\% fetal bovine serum (both Invitrogen; Thermo Fisher Scientific, Inc., Waltham, $\mathrm{MA}, \mathrm{USA}$ at $3^{\circ} \mathrm{C}$ in $5 \% \mathrm{CO}_{2}$.

Cell transfection. A PcDNA3.1/TNFR2-vector plasmid (Shanghai GenePharma Co., Ltd., Shanghai, China) was transfected into L428 cells using a Cell Line Nucleofector kit T (Amaxa; Lonza Group, Ltd., Basel, Switzerland) in order to upregulate TNFR2 expression; a GPU6/FAP-shRNA plasmid (Shanghai GenePharma Co., Ltd.) was transfected into Karpas299 cells to silence TNFR2 expression. Empty plasmids were used as controls, and transfection was performed according to the manufacturer's instructions. At $48 \mathrm{~h}$, the transfected cells were harvested for use in further procedures.

Reverse transcription-polymerase chain reaction (RT-PCR). Total RNA was extracted from cells using TRIzol (Invitrogen; Thermo Fisher Scientific, Inc.). cDNA was synthesized using a PrimeScript RT-PCR kit (Takara Biotechnology Co., Ltd., Dalian, China). All procedures were performed according to the manufacturer's protocols. PCR was performed using the kit and the results were analyzed by labwork software, version 4.0 (UVP, Upland, CA, USA). The primers were as follows: TNFR2: Forward, 5'-ACGTTCTCCAACACGACTTCATC-3'; Reverse, 5'-ATGATGACACAGTTCACCACTCC-3'. GAPDH: Forward, 5'-AGAAGGCTGGGGCTCATTTG-3' and Reverse, 5'-AGGGGCCATCCACAGTCTTC-3'. The experiment was performed 3 times.

Western blotting. Total protein was extracted and its concentration was measured using a bicinchoninic assay kit (Pierce; Thermo Fisher Scientific, Inc.) according to the manufacturer's protocol. A total of $300 \mu \mathrm{g}$ protein per lane was separated by $10 \%$ SDS-PAGE and transferred to a nitrocellulose membrane. After being Blocked using TBS with Tween 20 containing $5 \%$ non-fat dried milk for $1 \mathrm{~h}$ at room temperature, the membrane was incubated in primary antibody solutions, including antibodies against TNFR2 (cat. no. 19272-1-AP; dilution, 1:1,000; Proteintech Group, Inc., Wuhan Sanying Biotechnology, Wuhan, China), phospho-extracellular signal-related kinase 1/2 (ERK1/2), ERK1/2, phospho-Akt, Akt, $\beta$-catenin (cat. nos. 4370, 4695, 9611, 4691 and 8480, respectively; all dilution, 1:1,000; Cell Signaling Technology, Danvers, MA, USA) and GAPDH (cat. no. 13937-1-AP; dilution, 1:5,000; Proteintech Group, Inc.) at $4{ }^{\circ} \mathrm{C}$ overnight, followed by an peroxidase-linked goat anti-rabbit - IgG (cat. no. 4413; dilution, 1:4,000; Cell Signaling Technology) at room temperature for $1 \mathrm{~h}$. Immunoreactive signals were detected using an enhanced chemiluminescence reagent (Pierce; Thermo Fisher Scientific, Inc.).
Proliferation assay. Cells were counted and plated at $5 \times 10^{3}$ cells in 100- $\mu 1$ medium per well into a 96-well plate, in triplicate. At 24, 48, 72 and $96 \mathrm{~h}$ after plating, $10 \mu \mathrm{l}$ Cell Counting kit-8 (CCK-8; Beijing Solarbio Science \& Technology Co., Ltd., Beijing, China) solution was added. After $2 \mathrm{~h}$, the OD value was read at a wavelength of $450 \mathrm{~nm}$. The experiment was repeated 3 times.

Drug resistance assay. Cells were counted and plated into a 96-well plate in triplicate at $8 \times 10^{3}$ cells per well. At $24 \mathrm{~h}$, the medium was replaced with medium containing different concentrations of Adriamycin (ADM; HarveyBio, Inc., Beijing, China; 0.2, 0.4, 0.8, 1.6, 3.2 and $6.4 \mu \mathrm{mol} / \mathrm{l}$ ). Cells treated with $0 \mu \mathrm{mol} / 1$ served as the control. At $48 \mathrm{~h}$ later, $10 \mu \mathrm{l} \mathrm{CCK}-8$ solution was added. After $2 \mathrm{~h}$, the OD value was read at a wavelength of $450 \mathrm{~nm}$. Survival curves were constructed, and the $50 \%$ inhibitory concentration $\left(\mathrm{IC}_{50}\right)$ was calculated. Cell inhibition rate $=\mathrm{OD}$ value at each concentration of ADM/OD value at $0 \mu \mathrm{mol} / 1 \mathrm{ADM}(\%)$. To study potential molecular mechanism, AKT inhibitor LY294002 or WNT/ $\beta$-catenin inhibitor DKK1 was added into lymphoma cells $1 \mathrm{~h}$ before ADM and cells were cultured in this system for $48 \mathrm{~h}$. The final concentrations of LY294002 and DKK1 were $50 \mu \mathrm{M}$ and $50 \mathrm{ng} / \mathrm{ml}$ respectively. The experiment was repeated $\geq 3$ times.

Statistical analysis. All results are expressed as mean \pm standard deviation, and SPSS 13.0 software (SPSS, Inc., Chicago, IL, USA) was used for all statistical analysis. $\mathrm{IC}_{50}$ was calculated using regression analysis. The differences between groups were analyzed using two-tailed t tests. $\mathrm{P}<0.05$ was considered to indicate a statistically significant difference.

\section{Results}

TNFR2 expression is associated with the proliferation and drug resistance of lymphoma cells. As illustrated in Fig. 1, RT-PCR and western blot analysis confirmed the expression of TNFR2 in L428 and Karpas299 cells at the mRNA (Fig. 1A) and protein (Fig. 1B) levels. Karpas299 cells expressed relatively more TNFR2 and demonstrated a greater proliferative ability (Fig. 1C), and an increased resistance to ADM, with an $\mathrm{IC}_{50}$ of $1.42 \pm 0.24 \mu \mathrm{mol} / 1$ in Karpas 299 cells, compared with $0.728 \pm 0.15 \mu \mathrm{mol} / 1$ in L428 cells (Fig. 1D). This suggests that there may be a positive association between TNFR2 expression, and the proliferative and drug resistance abilities of lymphoma cells.

TNFR2 promotes the proliferation and drug resistance of lymphoma cells. To confirm whether TNFR2 affected the proliferation or contributed to the drug resistance of lymphoma cells, the expression of TNFR2 was decreased by transfection with shRNA against TNFR2, or increased by transfection with a TNFR2 plasmid. The shRNA-mediated knockdown in Karpas299 was verified at the mRNA and protein level (Fig. 2A and B; $\mathrm{P}<0.05$ ), in addition to the TNFR2 upregulation in L428 (Fig. 2C and D; P<0.05). The knockdown of TNFR2 in Karpas299 cells induced a significant decline in the proliferative ability (Fig. 2E) and a significant increase 
A

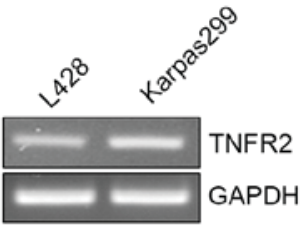

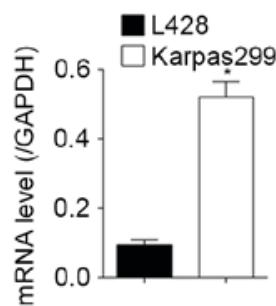

B

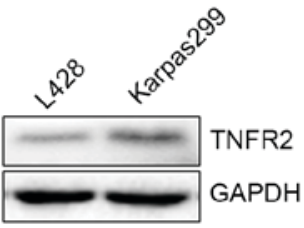

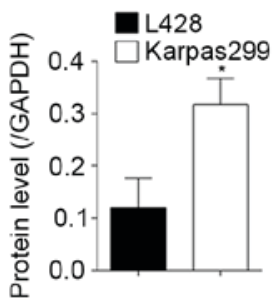

C

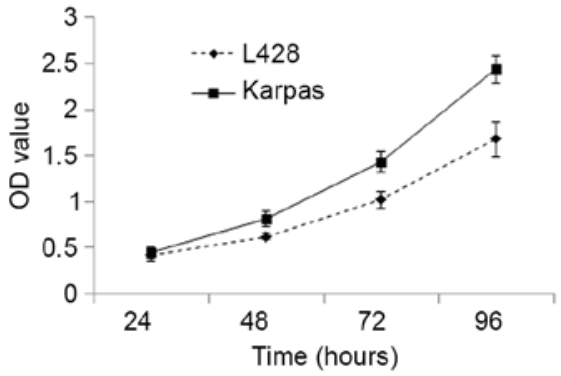

D

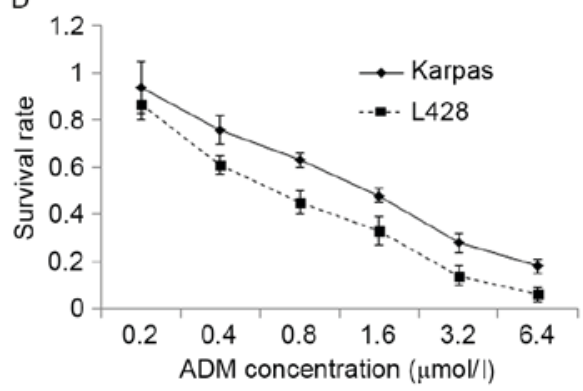

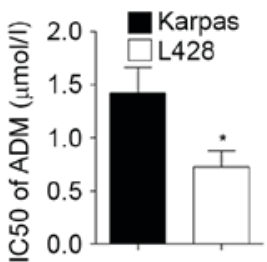

Figure 1. TNFR2 expression, proliferation and drug resistance of lymphoma cells. TNFR2 expression in L428 and Karpas299 cells was detected using (A) reverse transcription-polymerase chain reaction and (B) western blot analysis. (C) Cell proliferation and (D) drug resistance abilities were also determined ${ }^{*} \mathrm{P}<0.05$. TNFR2, tumor necrosis factor receptor 2; OD, optical density; ADM, adriamycin; $\mathrm{IC}_{50}, 50 \%$ inhibitory concentration.
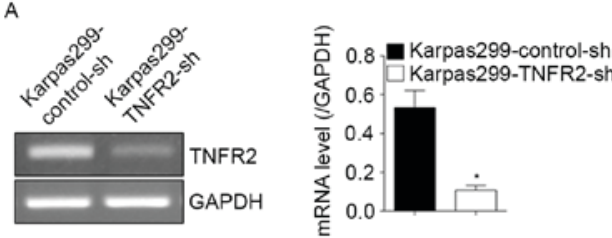

C

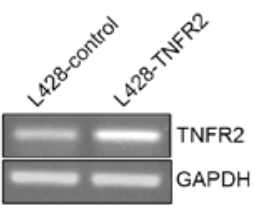

B
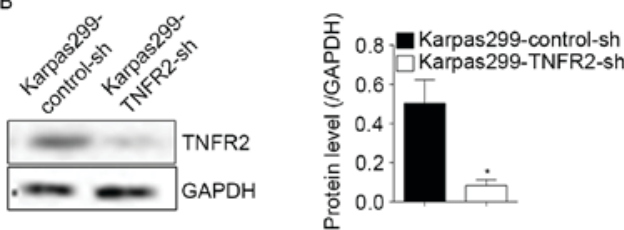

D

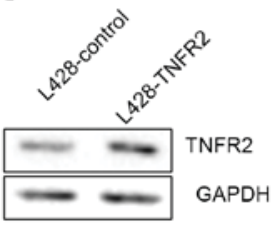

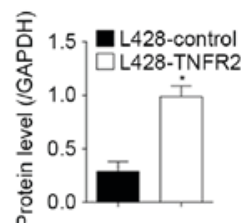

E

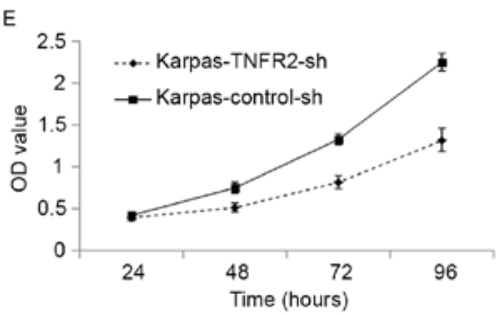

G

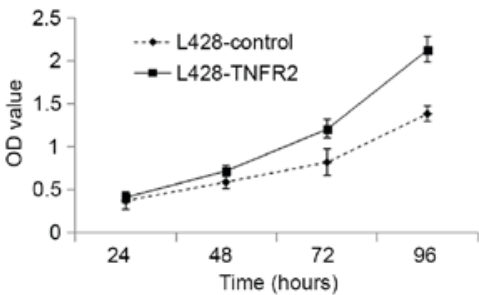

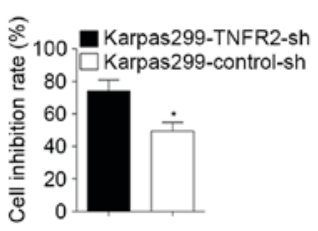

H

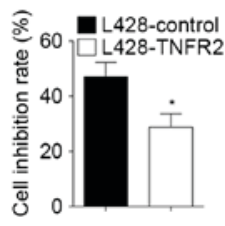

Figure 2. Effect of the downregulation or upregulation of TNFR2 in Karpas299 and L428 cells. Expression of TNFR2 was silenced in Karpas299 cells at the (A) mRNA and (B) protein levels following transfection with shRNA against TNFR2. The expression of TNFR2 was upregulated in L428 cells at the mRNA (C) and (D) protein levels following transfection with a plasmid containing TNFR2. (E) The proliferative ability of Karpas299 cells declined significantly following the silencing of TNFR2 and (F) the cell inhibition rate upon ADM treatment increased significantly. (G) The proliferative ability increased significantly following the upregulation of TNFR2 and $(\mathrm{H})$ the cell inhibition rate upon ADM treatment declined significantly. *P<0.05. TNFR2, tumor necrosis factor receptor 2; sh/shRNA, small hairpin RNA; ADM, adriamycin; OD, optical density. 


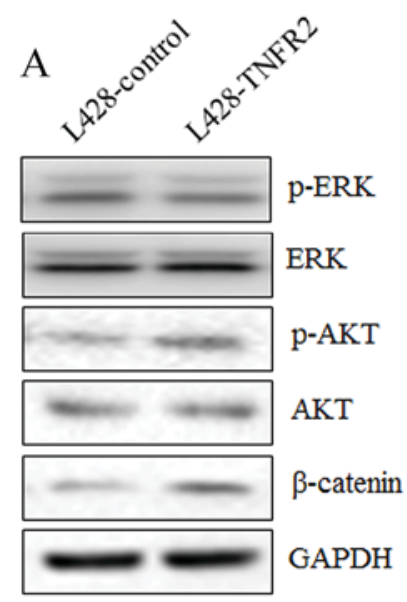

$\mathrm{B}$

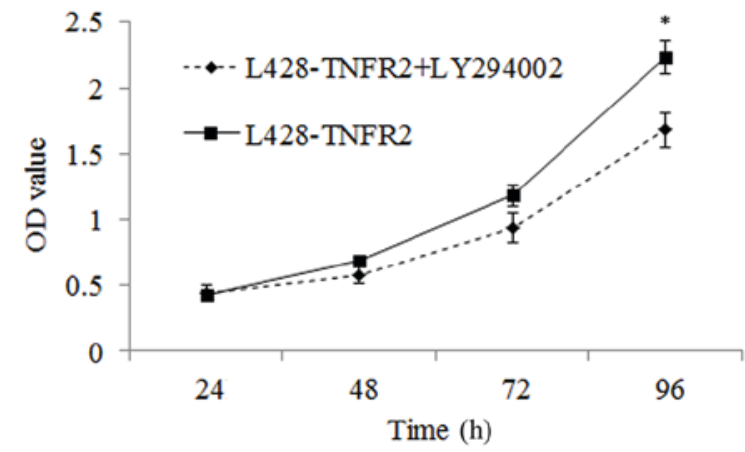

$\mathrm{C}$

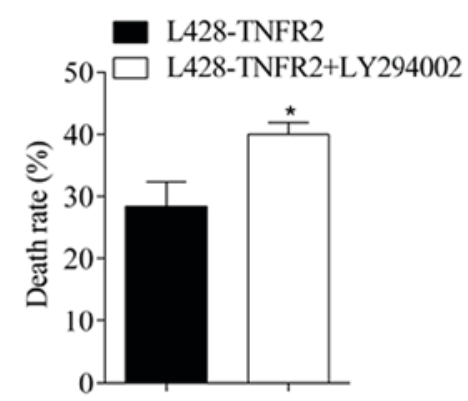

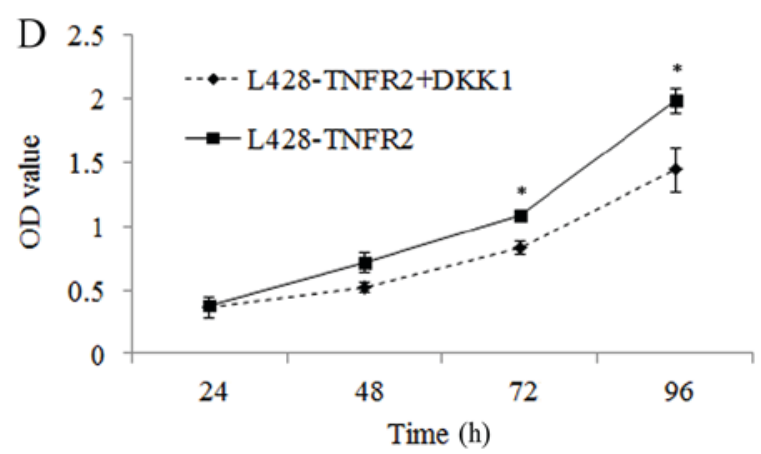

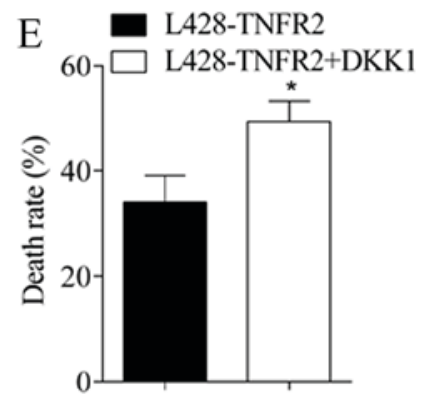

Figure 3. AKT and WNT/ $\beta$-catenin signaling function in the proliferation and drug resistance regulated by TNFR2. (A) $\beta$-catenin, p-AKT and p-ERK were detected by western blot following the upregulation of the expression of TNFR2 in L428 cells. The AKT inhibitor LY294002 (B) reduced the proliferation and (C) increased the cell inhibition rate of L248 cells. The WNT/ß-catenin blocker DKK1 (D) reduced the proliferation and (E) increased the cell inhibition rate in L248 cells. "P<0.05. TNFR2, tumor necrosis factor receptor 2; p-, phosphorylated; ERK, extracellular signal-regulated kinase; OD, optical density.

in the cell inhibition rate following ADM treatment, from $49.34 \pm 5.42 \%$ to $74.13 \pm 6.81 \%(\mathrm{P}<0.05$; Fig. $2 \mathrm{~F})$. Following the upregulation of TNFR2 expression in L428 cells, the proliferative ability increased significantly (Fig. 2G) and the cell inhibition rate upon ADM treatment declined from $47.03 \pm 5.25 \%$ to $28.71 \pm 4.90 \%(\mathrm{P}<0.05$; Fig. $2 \mathrm{H})$. These results confirmed the roles of TNFR2 in the proliferation and drug resistance of lymphoma cells.

AKT and WNT signaling pathways function in the regulation of proliferation and drug resistance of lymphoma cells by TNFR2. AKT, ERK and WNT/ $\beta$-catenin are important to the proliferation and drug resistance of tumor cells (12-14). To investigate the potential molecular mechanism underlying the effect of TNFR2 up- and downregulation, the expression of AKT, ERK and WNT/ $\beta$-catenin was examined. Following the upregulation of TNFR2 expression in L428 cells, the phosphorylation of $\mathrm{AKT}$ and the expression of $\beta$-catenin were significantly increased (Fig. 3A). No difference was observed in the phosphorylation of ERK. Following the addition of the AKT inhibitor, LY294002, the proliferation ability of L428 cells overexpressing TNFR2 declined significantly $(\mathrm{P}<0.05$ at $96 \mathrm{~h}$; Fig. 3B) and the cell inhibition rate following treatment with ADM increased from $28.38 \pm 3.94 \%$ to $40.03 \pm 1.88 \%(\mathrm{P}<0.05$; Fig. $3 \mathrm{C})$. On the application of a WNT/ $\beta$-catenin inhibitor, DKK 1 , the proliferative ability of L428 cells overexpressing TNFR2 also declined significantly
$(\mathrm{P}<0.05$ at 72 and $96 \mathrm{~h}$; Fig. 3D) and the cell inhibition rate following treatment with ADM increased from 34.07 $\pm 4.99 \%$ to $49.36 \pm 3.86 \%(\mathrm{P}<0.05$; Fig. $3 \mathrm{E})$. These results indicate that the AKT and $\mathrm{WNT} / \beta$-catenin signaling pathways contribute to the proliferative and drug resistance abilities of lymphoma cells, and that the mechanism is regulated by TNFR2.

\section{Discussion}

TNFR2 is encoded by the tumor necrosis factor receptor superfamily 1B (TNFRSF1B) gene (15). TNFR2 occurs as membrane binding TNFR2 (mTNFR2) and soluble TNFR2 (sTNFR2). To the best of our knowledge, the existing publications regarding TNFR2 in lymphoma are confined to the clinical significance of sTNFR2 in the blood. In 1998, Warzocha et al (16) reported that high levels of sTNFR2 were associated with clinical characteristics and a relatively poor prognosis for patients with Hodgkin's lymphoma. In 2012, Heemann et al (17) reported that patients with circulating levels of sTNFR2 $\geq 2.16 \mathrm{ng} / \mathrm{ml}$ exhibited a 2.07 -fold higher relative risk for a reduced overall survival time, and a 2.49-fold higher risk for reduced event-free survival time. In 2013, Nakamura et al (18) reported that high levels of sTNFR2 in the blood were associated with a relatively poor prognosis for patients with diffuse large B-cell lymphoma treated with the R-CHOP regimen. All of these studies consolidate a role 
for sTNFR2 in lymphoma. However, the role of TNFR2 in lymphoma cells remains uncharacterized.

ADM is a conventional chemotherapy drug currently used to treat lymphoma, with anti-tumor functions mediated by the inhibition of DNA synthesis $(19,20)$. In the present study, TNFR2 was expressed in L428 and Karpas299 cells; Karpas299 cells expressed relatively more TNFR2 and exhibited a greater proliferative ability and ADM resistance, indicating that TNFR 2 may be associated with the proliferation and drug resistance of lymphoma cells. Further experimentation revealed that TNFR2 overexpression enhanced the proliferation and drug resistance of L428 cells, whereas the silencing of TNFR2 in Karpas299 cells inhibited the proliferation and drug resistance of lymphoma cells. This confirms the role of TNFR2 in promoting lymphoma progression, and corroborates with the previous reports regarding circulating STNFR2 and prognosis for patients with lymphoma.

The AKT- and ERK-associated pathways are critical for mediating various physiological and pathological conditions. In tumors, their roles in proliferation, survival, adhesion, drug resistance and migration are well established $(12,13,21-23)$. It has been reported that inhibiting TNFR2 using a neutralizing antibody may block the activation of the AKT signal pathway in cholangiocarcinoma cells (9). The Wnt/ $\beta$-catenin signaling cascade is considered to be central to carcinogenesis; it can affect a number of traits associated with cell malignancy, including proliferation, migration, drug resistance and even the maintenance of stemness (14,24). In the present study, it was demonstrated that TNFR 2 could function in proliferation and drug resistance via the $\mathrm{AKT}$ and $\mathrm{WNT} / \beta$-catenin signaling pathways. This is consistent with the roles of AKT, WNT/ $\beta$-catenin and TNFR2 in tumor progression that have been reported in the past. However, the question remains of whether the drug resistance role of TNFR 2 could be partly or wholly attributed to its pro-proliferative role. Further in vitro experimentation will be required to answer this question.

In conclusion, TNFR2 promoted proliferation and drug resistance via AKT and $\mathrm{WNT} / \beta$-catenin signaling pathways in lymphoma cells. This may contribute to the understanding of TNFR2 function in lymphoma, and provides a basis for discovering novel therapeutic targets against lymphoma.

\section{Acknowledgements}

Not applicable.

\section{Funding}

No funding was received.

\section{Availability of data and materials}

All data generated or analyzed during the present study are included in this published article.

\section{Authors' contributions}

YL performed cell experiments and revised the present study. YW revised the present study. MX performed cell experiments. YH performed cell experiments. YZ performed data analysis. SL designed the present study and wrote the present paper.

\section{Ethics approval and consent to participate}

Not applicable.

\section{Consent for publication}

Not applicable.

\section{Competing interests}

The authors declare that they have no competing interests.

\section{References}

1. Kewitz S, Kurch L, Volkmer I and Staege MS: Stimulation of the hypoxia pathway modulates chemotherapy resistance in Hodgkin's lymphoma cells. Tumour Biol 37: 8229-8237, 2016.

2. Fino P, Spagnoli AM, Ruggieri M, Marcasciano M and Scuderi N: Bilateral hand squamous-cells carcinoma in patient affected with non-Hodgkin lymphoma. Case report and literature review. G Chir 36: 172-182, 2015.

3. Hu RH, Sun WL, Zhao H, Hui WH, Guo YX, Wan SG and Su L: Rituximab combined with EPOCH regimen for treatment of diffuse large $\mathrm{B}$ cell lymphoma of the gastrointestinal tract: Analysis of 4 cases. Nan Fang Yi Ke Da Xue Xue Bao 36: 1291-1294, 2016 (In Chinese).

4. Khandelwal A, Trinkaus MA, Ghaffar H, Jothy S and Goldstein MB: A case report of unusually long lag time between immunotactoid glomerulopathy (itg) diagnosis and diffuse large B-cell lymphoma (DLBCL) development. BMC Nephrol 17: 140, 2016.

5. Yang D, Fu X, Zhang X, Li W and Zhang M: Therapy-related acute myeloid leukemia in patients with lymphoma: A report of four cases and review of the literature. Oncol Lett 10: 3261-3265, 2015.

6. Tang W, Lu Y, Tian QY, Zhang Y, Guo FJ, Liu GY, Syed NM, Lai Y, Lin EA, Kong L, et al: The growth factor progranulin binds to TNF receptors and is therapeutic against inflammatory arthritis in mice. Science 332: 478-484, 2011.

7. Guo Z, Li Q, Han Y, Liang Y, Xu Z and Ren T: Prevention of LPS-induced acute lung injury in mice by progranulin. Mediators Inflamm 2012: 540794, 2012.

8. Zhao YP, Tian QY, Frenkel S and Liu CJ: The promotion of bone healing by progranulin, a downstream molecule of BMP-2, through interacting with TNF/TNFR signaling. Biomaterials 34 : 6412-6421, 2013.

9. Tanimura Y, Kokuryo T, Tsunoda N, Yamazaki Y, Oda K, Nimura Y, Naing Mon N, Huang P, Nakanuma Y, Chen MF, et al: Tumor necrosis factor alpha promotes invasiveness of cholangiocarcinoma cells via its receptor, TNFR2. Cancer Lett 219: 205-213, 2005.

10. Johrer K, Janke K, Krugmann J, Fiegl M and Greil R: Transendothelial migration of myeloma cells is increased by tumor necrosis factor (TNF)-alpha via TNF receptor 2 and autocrine up-regulation of MCP-1. Clin Cancer Res 10: 1901-1910, 2004.

11. Minich SS: Brentuximab vedotin: A new age in the treatment of hodgkin lymphoma and anaplastic large cell lymphoma. Ann Pharmacother 46: 377-383, 2012.

12. Dahlmann M, Okhrimenko A, Marcinkowski P, Osterland M, Herrmann P, Smith J, Heizmann CW, Schlag PM and Stein U: RAGE mediates S100A4-induced cell motility via MAPK/ERK and hypoxia signaling and is a prognostic biomarker for human colorectal cancer metastasis. Oncotarget 5: 3220-3233, 2014.

13. Miao B and Degterev A: Targeting phospshatidylinositol 3-kinase signaling with novel phosphatidylinositol 3,4,5-triphosphate antagonists. Autophagy 7: 650-651, 2011.

14. Sebio A, Kahn M and Lenz HJ: The potential of targeting Wnt $/ \beta$-catenin in colon cancer. Expert Opin Ther Targets 18: 611-615, 2014.

15. Xu F, Zhou G, Han S, Yuan W, Chen S, Fu Z, Li D, Zhang H, Li D and Pang D: Association of TNF- $\alpha$, TNFRSF1A and TNFRSF1B gene polymorphisms with the risk of sporadic breast cancer in northeast Chinese han women. PLoS One 9: e101138, 2014. 
16. Warzocha K, Bienvenu J, Ribeiro P, Moullet I, Dumontet C, Neidhardt-Berard EM, Coiffier B and Salles G: Plasma levels of tumour necrosis factor and its soluble receptors correlate with clinical features and outcome of Hodgkin's disease patients. Br J Cancer 77: 2357-2362, 1998

17. Heemann C, Kreuz M, Stoller I, Schoof N, von Bonin F, Ziepert M, Löffler M, Jung W, Pfreundschuh M, Trümper L and Kube D: Circulating levels of TNF receptor II are prognostic for patients with peripheral T-cell non-Hodgkin lymphoma. Clin Cancer Res 18: 3637-3647, 2012.

18. Nakamura N, Goto N, Tsurumi H, Takemura M, Kanemura N, Kasahara S, Hara T, Yasuda I, Shimizu M, Sawada M, et al: Serum level of soluble tumor necrosis factor receptor 2 is associated with the outcome of patients with diffuse large B-cell lymphoma treated with the R-CHOP regimen. Eur J Haematol 91: 322-331, 2013.

19. Engert A: ABVD or BEACOPP for advanced Hodgkin lymphoma. J Clin Oncol 34: 1167-1169, 2016.

20. Ajish JK, Ajish Kumar KS, Chattopadhyay S and Kumar M: Glycopolymeric gel stabilized N-succinyl chitosan beads for controlled doxorubicin delivery. Carbohydr Polym 144: 98-105, 2016
21. Hu SY, Tai CC, Li YH and Wu JL: Progranulin compensates for blocked IGF-1 signaling to promote myotube hypertrophy in $\mathrm{C} 2 \mathrm{C} 12$ myoblasts via the PI3K/Akt/mTOR pathway. FEBS Lett 586: 3485-3492, 2012.

22. Bai T, Lian LH, Wu YL, Wan Y and Nan JX: Thymoquinone attenuates liver fibrosis via PI3K and TLR4 signaling pathways in activated hepatic stellate cells. Int Immunopharmacol 15: 275-281, 2013.

23. Li N, Cui J, Duan X, Chen H and Fan F: Suppression of type I collagen expression by miR-29b via PI3K, Akt, and Sp1 pathway in human Tenon's fibroblasts. Invest Ophthalmol Vis Sci 53: 1670-1678, 2012.

24. Sawa M, Masuda M and Yamada T: Targeting the Wnt signaling pathway in colorectal cancer. Expert Opin Ther Targets 20: 419-429, 2016. 